\title{
La responsabilité
}

\section{Véra Jacob de Fradera}

\author{
Docteur en Droit, Université Paris II \\ Professeur à l'Université Fédérale du Rio Grande do Sull
}

\section{Introduction}

Comme nous le savons tous, la responsabilité du médecin est de nature contractuelle, fondée sur la faute.

Pour ce qui est de la nature de l'obligation du médecin, la majeure partie de la doctrine admet la distinction entre obligations de moyens et de résultat, découverte par $\mathrm{M}$. Demogue, répandue partout grâce aux écrits de MM. Henri Mazeaud et André Tunc².

La doctrine et la jurisprudence brésiliennes ont subi, après l' Indépendence du Portugal, une forte influence de la doctrine française dans tous les domaines du droit. Cette attirance pour la littérature juridique française s 'explique par la connaissance de la langue française par les milieux cultivés et scientifiques de la societé brésilienne et par une présence de la France dans toutes les manifestations culturelles du pays, comme par exemple, la mode, la musique, l'art en général.

\footnotetext{
${ }^{1}$ Capitulo de livro intitulado La responsabilité, Aspects Nouveaux, Travaux de l'Association Henri Capitant, Jourriées panaméennes, tome L/1999, p. 217 / 220. Ed. LGDJ, ISBN: 2-275-02286-4, 2003, Paris.

${ }^{2}$ M.Henri Mazeaud, «Essai de classification des obligations: obligations contractuelles et extracontractuelles, obligations déterminées et obligations générales de prudence et diligence ", in RTDciv. 1936, p.1 et s. n.50; TUNC, André, "La distinction des obligations de réseultat et des obligations de diligence ", JC 45, éd. G, I, 449.
} 
Le droit civil brésilien est un amalgame d'influences très variées (Les Ordonnances du Portugal, le Droit Canon, le Droit Romain, les Pandectes) mais, dans le domaine de la responsabilité civile, c'est l'influence française qui est la plus remarquable et le législateur brésilien a même reproduit dans le Code Civil , l'atticle 1382 du Code Napoléon.

Ainsi, la doctrine et la jutisprudence brésiliennes sont d'accord pout qualifier l'obligation contractuelle du médecin comme une obligation de moyens, c'est-à-dite que le médecin doit déployer tous ses efforts pour guérir le malade, mais il n'est pas obligé d'obtenir la guétison, car on reconnait que plusieurs facteurs interviennent ; par ailleurs, il y a toujouts des maladies dont l'origine est inconnue ou pour lesquelles une thérapie capable de les maîtriset n'a pas encore été ttouvée. Il existe toujours un aléa dans ce contrat.

En conséquence, le médecin n' est responsable civilement que s'il a manqué à ses devoirs professionnels envers le malade: c'est-à-dire qu' il n'a pas employé toute sa diligence et ses connaisances, tous ses efforts, pout le guérit.

Mais c'est au patient de prouver que le médecin a manqué à ses obligations.

Pat ailleurs, l 'obligation du médecin peut être de résultat, ou déterminée, quand le débiteur (le médecin) doit atteindre un résultat. C’est par exemple, le cas du médecin radiologue, du biocbimiste et du chirurgien plasticien.

En ce qui concerne la charge de la preuve, dans ce cas, c'est le médecin qui doir prouver que l'inexécution est dûe à une cause quil lui est étrangète.

L'obligation du médecin, dans certaines spécialités médicales, comme la chirurgie esthétique, est considétée depuis longtemps, comme de résultat.

Aujourd'hui la docrrine et la jurisprudence commencent cependant à admettre quelques dérogations à cette tègle. Pour ce qui est de ce domaine, le droit brésilien présente quelques particularités.

Nous allons donc, dans un premier temps, nous occuper de la position dominante dans la doctrine et la jurisprudence brésiliennes pour ce qui est de la nature de l'obligation du médecin plasticien, quand la chirurgie est purement esthétique;

Ensuite, notre étude portera sur quelques particularités de la responsabilité civile du chirurgien plasticien en droit brésilien.

I. L'obligation du chirurgien plasticien est une obligation de résultat, quand la chirurgie revêt un caractère purement esthétique

Sous l'influence de la doctrine française qui, pendant longtemps, a considéré l'obligation du chirutgien plasticien comme une obligation de résultat, la doctrine majoritaire, ainsi que les tribunaux brésiliens, ont adopté ce même critère. 
En effet, la personne qui va chez le chirurgien plasticien pour se faire opérer de son nez qu' elle trouve trop grand ou qui veut enlever quelques rides autour de ses yeux, n'est pas malade, c'est seulement un être sain qui désire avoír une apparence plus jeune ou prétend corriger un de ses traits. Dans ce cas, le médecin a, selon cette conception du problème, une obligation de résultat, il doit obtenir le résultat qu'il a promis à son client. Le cas écheant, il est responsable civilement. C'est au médecin de prouver que si le résultat n'a pas été atteint à cause d'une autre raison, la force majeure, par exemple.

La doctrine brésilienne dominante adopte cette qualification en ce qui concerne la chinurgie purement esthétique. Dans ce sens, on peut citer MM. Aguiar Dias ${ }^{3}$, Caio Mário da Silva Pereira ${ }^{4}$ et Genival Velloso França ${ }^{s}$.

Tous ces auteurs remarquent que les Tribunaux sont très sévères avec les médecins plasticiens et qu'ils rendent plus lourdes leurs obligations. Un exemple: l'obligation d'informer porte sur tous les risques, les plus courants mais aussi les risques exceptionnels.

M. Velloso França, dans son livre Direito Médico, écrit ainsi qque la chirurgie purement esthétique devrait être écartée des domaines de la Médecine, parce que cette spécialité n’a pas des objectifs curatifs, et elle vise des buts souvent inaccessibles, par exemple, rajeunir de 30 ans une personne agée de 80 !

Les arrêts sont très nombreux, et tous les tribunaux nationaux jugent pareillement les cas de responsabilité civile du médecin plasticien: le Supérieur Tribunal de Justice (STJ) à Brasilia er les Tribunaux des États membres de la Fédération.

Cependant, la doctrine et la jurisprudence brésiliennes classifient comme de résultat l'obligation du chirurgien quand la chirurgie est purement esthétique et de moyens quand la chirurgie plastique est réparatrice.

Dans le même sens, M. Miguel KFOURI NETO ${ }^{6}$ auteur d'une monographie sur la Responsabilité Civile du Médecin adopte la thèse selon laquelle il faut distinguer entre Chirurgie Esthétique stricto sensu, c'est-à dire celle dont les buts sont purement esthétiques, et la Chirurgie Esthétique réparatrice. Dans le premier cas, lobligation du chirurgien est de résultat, dans le second, de moyens Tous les 'Tribunaux sont d'accord sur cette distinction.

Nous allons à présent préciser qu'il existe cependant une partie de la doctrine brésilienne er quelques décisions des tribunaux, qui considèrent, en matière de chirurgie esthétique, l'obligation du médecin plasticien n'est qu'une obligation de moyens.

\footnotetext{
${ }^{3}$ Aguiar Dias, Da Responsabilidade civil, v. I, Ed. Forense, Rio de Janeiro, 1979 p. 303.

${ }^{4}$ Caio Mário da Silva Pereira, Responsabilidade Civil, Ed. Forense, Rio de Janeiro, 1993, p.168 ets.

${ }^{5}$ Genival , Velloso França, Direito Médico, Fundo Editorial BYK-Procienx, 1994, p. 142.

"Miguel Kfouri Neto, Responsabilidade Civil do Médico, 3a. ed. Ed. RT, 1998 , p. 166 ets .
} 


\section{L'obligation du chirurgien plasticien doit être considérée comme une} obligation de moyens selon quelques juristes brésiliens, même quand la chirurgie revêt un caractère purement esthétique

Quelques auteurs de la doctrine brésilienne considèrent cependant l'obligation du chirurgien esthétique comme de moyens, car, selon ces auteurs il faudrait prendre en compte l'ensemble des qualités de chaque patient, par exemple, son âge, sa peau, le fait qu'il fume encore ou ait fumé, etc. Pour ces juristes, il s'agit du même aléa que celui qui est inhérent aux autres spécialités médicales. Ce courant doctrinal, malgré l'importance des auteurs qui l' adoptent, n'a pas encore reçu la consécration des juges er des tribunaux.

Parmi les auteurs qui essaient de diffuser cette idée, on peut citer le Ministre du Supérieur Tribunal Fédéral, M. Ruy Rosado de Aguiar ?, pour qui, en dépit de ce que la doctrine et la jurisprudence majoritaires qualifient de résultat l' obligation du chirurgien plasticien, la vérité appartient à ceux qui attribuent au médecin plasticien une obligation de moyens. Les auteurs qui adoptent cette conception ont subi l'influence française.

Cette vision est assez récente parmi nous, et elle remonte à la publication d'un artêt de la 1ère. Chambre Civile de la Cour de Cassation ${ }^{8}$, commenté par François Chabas, et à un article de doctrine, dont l'auteur Luis Andorno de l'Université de Rosário, Republique argentine, est fortement influencé par les juristes français.

M. Chabas affirme dans son commentaire que «toute intervention sur le corps bumain est aléatoire, les réactions du corps étant imprévisibles. Nul ne peut prévoir par exemple comment se fera une cicatrisation ... À tel point que nous pensons que serait ipso facto en faute le plasticien qui promettrait un résultat, parce qu'il ne pourrait ne pas être conscient $d u$ caratère falacieux de son engagement. .

Depuis cet arrêt, on observe un changement, pour l'beure encore assez timide, dans la jurisprudence brésilienne rendue en matière de responsabilité du cbirugien plasticien.

Deux exemples jurisprudentiels de cette nouvelle tendance peuvent à cet égard être mentionnés:

- Tribunal de Justice de l'Etat du Paraná, recours nº 34834 -1, Juge: Abrahão Miguel, 20.6.1995: rendu en matiére de chirurgie esthétique, cette decision qualifie l'obligation de moyens, décidant que c'est à la victime de prouver la faute du chirurgien;

\footnotetext{
7Ruy Rosado de Aguiar, "Responsabilidade Civil do Médico», in Revista dos Tribunats - 718, agosto 1995, p.33 ets.

${ }^{8}$ Responsabilité civile - médecin. Chirurgien esthétique. a) obligation de moyens. Prothèses Mamaires. Résultats aléatoires. Faute (non). b) Obligation d'information. Manquement. Preuve. Charge. Patient: JCP $\mathrm{n} 1993, \mathrm{n}^{\circ} 25$.

"Luis Andorno, "La responsabilidad civil médica " in Revista da AJURIS, 59, p.224, Porto Alegre, Brasil,
} 
- Tribunal de Justice de l'Etat du Rio Grande do Sul; appel n ${ }^{\circ} 597083948,18.9 .19975$ ème Chambre Civile.

Un des juges ${ }^{10}$ a indiqué aux membres de la Chambte que l'obligation du chirurgien plasticien, même s'il s'agit d'une chirurgie purement esthétique, était de moyens, non de résultat. Dans cette Chambre, il a été le seul a avoir certe conception.

\section{Conclusion}

Il apparaît à la lumière de cet exposé que l'influence de la doctrine et de la jurisprudence françaises sont roujours remarquables dans le domaine de la responsabilité civile au Brésil.

10 M. Alfredo Guilherme Englert. 